Case Report

\title{
Idiopathic Retroperitoneal Fibrosis: A Case of Women 75-years Old Without Ureteral Involvement
}

\author{
Foieni F ${ }^{1}$, Liedke A ${ }^{2}$, Spinedi $L^{3}$, Monotti $R^{2}$ \\ ${ }^{1}$ Internal Medicine, Busto Arsizio Hospital, ASST Valle Olona, v. Arnaldo da Brescia 1, 21052 Busto Arsizio, \\ Varese, Italy \\ 2 Internal Medicine, Locarno Hospital, EOC, v. Ospedale 1, 6600 Locarno, Switzerland \\ ${ }^{3}$ Angiology Service, Locarno Hospital, EOC, v. Ospedale 1, 6600 Locarno, Switzerland \\ *Correspondence: fabrizio.foieni@ asst-valleolona.it
}

\begin{abstract}
A 75-year-old female is referred for evaluation of a progressive leg swelling. Angiological and MRI evaluation show the presence of inflammatory tissue surrounding the aorta and the left iliac vessels, causing significant stenosis. Upon suspicion of retroperitoneal fibrosis, we actively seek for secondary causes such as malignancies, autoimmune diseases and infections, as well as IgG4-related disease. All investigations result negative and we make the diagnosis of idiopathic retroperitoneal fibrosis. Steroid therapy was started and we observed a rapid amelioration of symptoms and radiological disappearance of the inflammatory tissue around the aorta.
\end{abstract}

Keywords: ANCA; Hashimoto; Hydronephrosis; IgG4; Periaortitis; Retroperitoneal fibrosis; Vasculitis

How to cite this paper: Foieni, F.,

Liedke, A., Spinedi, L., \& Monotti, R. (2022). Idiopathic Retroperitoneal Fibrosis: A Case of Women 75-years Old Without Ureteral Involvement. Open Journal of Medical Sciences, 2(1), 1-4. Retrieved from https://www.scipublications.com/journal/index.php/ojms/article/view/197

Received: November 02, 2021 Accepted: December 12, 2021 Published: December 13, 2021

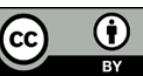

Copyright:@ 2022 by the authors. Submitted for possible open access publication under the terms and conditions of the Creative Commons Attribution (CC BY) license (http://creativecommons.org/licenses /by/4.0/).

\section{Introduction}

Idiopathic retroperitoneal fibrosis is a rare and very heterogeneous disease, recent reports suggest an increasing incidence possibly due to improvement in detection methods [5]. We show that it can present itself without any symptoms but isolated leg swelling (without elevation of inflammatory parameters, abdominal and renal symptoms). It is important to rule out secondary causes of RPF such as malignancies, infections, drugs, previous medical interventions.

\section{Case Presentation and follow up}

A-75-year-old female was referred for angiologic evaluation for a 3-week history of left leg swelling. It started as a mild perimalleolar swelling and it slowly progressed to the whole leg. The patient complains of heaviness and mild burning especially at rest, but there is no pain associated. There is no history of trauma or other apparent trigger. Medical history is significant for hypertension and untreated psoriasis vulgaris; the patient is a smoker (25py). Current medication consists of Valsartan, Hydrochlorothiazid and Pantoprazol.

During medical examination, we find a clinically relevant swelling of the leg ( $>7 \mathrm{~mm}$ difference at calf level), an ecodoppler of the left lower limb shows an obstruction of the left external iliac vein due to a possible external compression with some enlarged inguinal lymph nodes (1-3 cm diameter). A CT scan is therefore requested; it shows the presence of a solid tissue located anterior to the aorta before the iliac bifurcation (L4-L5 height) that extends downward to envelop the left iliac vessels. This finding is further investigated by angio-RM, which confirms the solid tissue around the aorta presenting enhancement after contrast medium; such presentation is compatible with inflammatory 
tissue. Such tissue is also present around the medial third of the left external iliac vein, producing significant stenosis thus hindering venous return(See Figure 1).

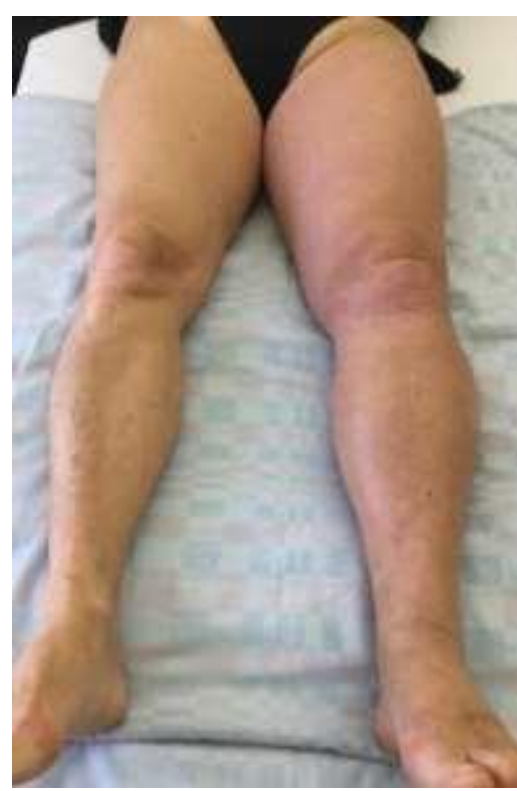

Figure 1. Patient at the presentation (first medical examination)

\section{Discussion}

We are therefore confronted with a picture of periaortitis, the origin of which is still to be determined. The most recent literature tells us that, in the absence of aneurysmatic

dilatation of the aorta, this type of inflammatory tissue can be interpreted as a form of retroperitoneal fibrosis [1-4].

Retroperitoneal fibrosis can be either idiopathic or be associated with other inflammatory or autoimmune diseases, malignancies, infections, a wide range of drugs including ergot derivatives, dopamine agonists, methyldopa, beta-blockers, hydralazine, non steroideal anti-inflammatory drugs, trauma or previous medical interventions such as radiotherapy or surgery in the adjacent area [1-4]. Recent studies suggest that many cases of idiopathic retroperitoneal fibrosis are associated with IgG4-related disease [6]. In our case, measurement of $\mathrm{IgG} 4$ in peripheral blood results within normal range and we didn't perform the histopathological examination due to the patient's non-compliance with the surgery. We must also actively seek for secondary causes. Routine blood tests are unremarkable, there is no elevation of inflammatory parameters. Serum electrophoresis is unremarkable, immunophenotypization of peripheral blood shows no evidence of lymphoproliferative disease. A full gynecological search for neoplasia is unremarkable, as well as an ano-rectal investigation. A temporal artery biopsy does not provide evidence for gigantocellular arteritis. An extended autoimmune panel is negative, serologic search for tubercolosis, HIV, C. burnetii, Brucella, Bartonella henselae, Bartonella quintana and T. pallidum infection all came back negative, as well as serial blood cultures.

High dose steroid therapy is therefore initiated $(60 \mathrm{mg}, 1 \mathrm{mg} / \mathrm{kg}$ daily), along with anticoagulant therapy with Xarelto $10 \mathrm{mg}$ daily for thrombosis prevention and osteoporosis prophylaxis with vitamin $\mathrm{D}$ and bisphosphonate therapy. We also prescribe manual lymph drainage massage and compressive dressing of the limb.(See Figure 2 and Figure 3) 


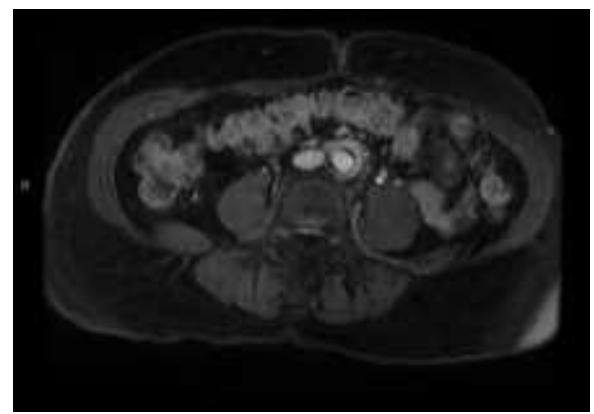

Figure 2. Abdominal MRI. Before steroid therapy.

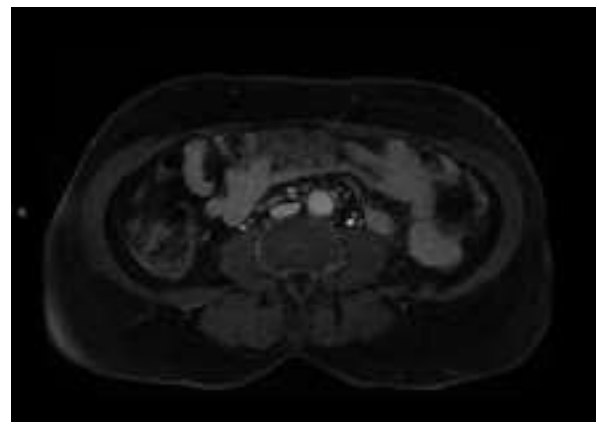

Figure 3. Abdominal MRI. After six months of therapy.

A follow up angiologic evaluation after 1 month shows a net reduction of the swelling, ecocolordoppler examination shows a clear reduction of the iliac vein stenosis but persistent enhancement of the tissue surrounding the aorta after intravenous infusion of ultrasound contrast medium. Steroid therapy is reduced at $40 \mathrm{mg} /$ daily considering the clinical improvement and due to mild side effects.

Follow up MRI is performed 1 month later, which shows a complete resolution of the external iliac vein stenosis, the inflammatory tissue surrounding the aorta is not detected anymore. Steroid therapy is thus reduced at $30 \mathrm{mg}$ daily. Anticoagulant therapy is suspended considering the complete resolution of the vein stenosis.

6 months later, the patient has been reviewed and shows no signs of illness. We have repeated an ecocolordoppler and a control RMI that have shown a persistent complete resolution of the peri-iliac fibrotic picture.

\section{Conclusion}

We discussed about a case of retroperitoneal fibrosis that presented itself only as an isolated leg swelling without systemic symptoms and without elevation of inflammatory parameters in peripheral blood. Fortunatly, we have not encountered any complications about die pielic and ureteral system. Steroid therapy was started and a prompt amelioration of symptoms and gradual disappearance of the inflammatory tissue upon MRI examination was found. Therapy was continued for a total of 6 months.

Glucocorticoids are the mainstay of therapy, although adjunctive immunosuppressive agents are used in relapsing patients [7-9]

\section{Authors' Contributions}

Foieni F. contributed to the acquisition of data, clinical followup and editing the manuscript. Liedke A. contributed to acquisition of data and editing the manuscript. Spinedi L. contributed to clinical evaluation and ultrasound controls. Monotti R. contributed to editing the manuscript. 
No financial relationships relevant to this article

\section{Informed Consent}

The patient gave full consent for publishing this article including pictures.

\section{Conflicts of Interest}

All the authors declare no conflict of interest regarding this case.

\section{References}

[1] Gómez Rivas J, Quintana LM, Álvarez-Maestro M, Aguilera A, Martinez Piñeiro L, Sarikaya S. Retroperitoneal fibrosis: A literature review. Arch Esp Urol. 2020 Jan;73(1):60-67. English, Spanish. PMID: 31950925.

[2] Conard J, Mancini M. Retroperitoneal Fibrosis. J Am Osteopath Assoc. 2017 May 1;117(5):340. doi: 10.7556/jaoa.2017.067. PMID: 28459474.

[3] Rossi GM, Rocco R, Accorsi Buttini E, Marvisi C, Vaglio A. Idiopathic retroperitoneal fibrosis and its overlap with IgG4-related disease. Intern Emerg Med. 2017 Apr;12(3):287-299. doi: 10.1007/s11739-016-1599-z. Epub 2017 Jan 9. PMID: 28070877.

[4] Vaglio A, Maritati F. Idiopathic Retroperitoneal Fibrosis. J Am Soc Nephrol. 2016 Jul;27(7):1880-9. doi: 10.1681/ASN.2015101110. Epub 2016 Feb 9. PMID: 26860343; PMCID: PMC4926988.

[5] Runowska M, Majewski D, Puszczewicz M. Retroperitoneal fibrosis - the state- of-the-art. Reumatologia/Rheumatology. 2016;54(5):256-263. doi:10.5114/reum.2016.63667.

[6] Pieringer, H., Parzer, I., Wöhrer, A. et al. IgG4- related disease: an orphan disease with many faces. Orphanet J Rare Dis 9, 110 (2014). https://doi.org/10.1186/s13023-014-0110-z

[7] Brandt AS, Kamper L, Kukuk S, et al. Associated findings and complications of retroperitoneal fibrosis in 204 patients: Results of a urological registry. J Urol 2011; 185: 526-531

[8] Khosroshahi A, Carruthers MN, Stone JH, et al. Rethinking Ormond's disease. Medicine (Baltimore) 2013; 92 : 82-91.

[9] Zen Y, Onodera M, Inoue D, et al. Retroperitoneal fibrosis: a clinicopathologic study with respect to immunoglobulin G4.Am J Surg Pathol 2009; 33: 1833-1839 